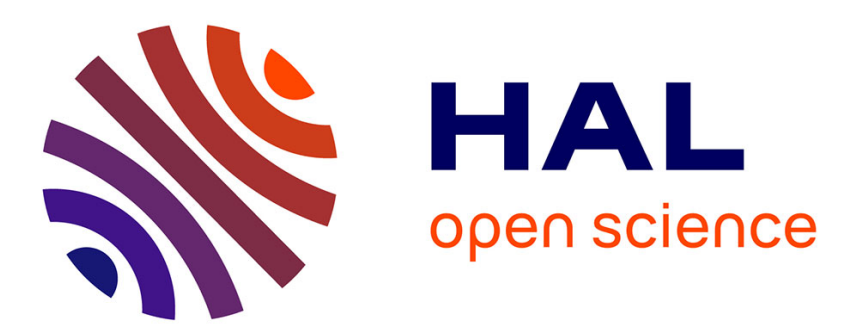

\title{
Calculation of the circular dichroism spectra of carbon monoxy- and deoxy myoglobin: interpretation of a time-resolved circular dichroism experiment.
}

\author{
Thibault Dartigalongue, François Hache
}

\section{- To cite this version:}

Thibault Dartigalongue, François Hache. Calculation of the circular dichroism spectra of carbon monoxy- and deoxy myoglobin: interpretation of a time-resolved circular dichroism experiment.. Journal of Chemical Physics, 2005, 123 (18), pp.184901. 10.1063/1.2041467 . hal-00827924

HAL Id: hal-00827924

https://hal-polytechnique.archives-ouvertes.fr/hal-00827924

Submitted on 15 May 2014

HAL is a multi-disciplinary open access archive for the deposit and dissemination of scientific research documents, whether they are published or not. The documents may come from teaching and research institutions in France or abroad, or from public or private research centers.
L'archive ouverte pluridisciplinaire HAL, est destinée au dépôt et à la diffusion de documents scientifiques de niveau recherche, publiés ou non, émanant des établissements d'enseignement et de recherche français ou étrangers, des laboratoires publics ou privés. 


\title{
Calculation of the circular dichroism spectra of carbon monoxy- and deoxy myoglobin: Interpretation of a time-resolved circular dichroism experiment
}

\author{
Thibault Dartigalongue and François Hache ${ }^{\mathrm{a})}$ \\ Laboratoire d'Optique et Biosciences-Centre National de la Recherche Scientifique (CNRS)-Institute \\ National de la Sante et de la Recherche Medicale (INSERM), Ecole Polytechnique, \\ 91128 Palaiseau Cedex, France
}

(Received 20 June 2005; accepted 28 July 2005; published online 4 November 2005)

\begin{abstract}
A calculation of the circular dichroism (CD) spectra of carbon monoxy- and deoxy myoglobin is carried out in relation with a time-resolved CD experiment. The calculation is based on the polarizability theory and the parameters are adjusted to fit the experimental absorption and $\mathrm{CD}$ spectra. By performing the calculation for intermediate configurations of the protein, we are able to propose an explanation of the CD structure observed on a sub-100 ps time scale. The role of the proximal histidine is, in particular, clearly demonstrated in the first step of the myoglobin relaxation from its liganded to it deliganded form. (c) 2005 American Institute of Physics.
\end{abstract}

[DOI: $10.1063 / 1.2041467$ ]

\section{INTRODUCTION}

Conformational changes that occur in carbon monoxy myoglobin $(\mathrm{MbCO})$ are known to play an important physiological role as a model of the trigger of the allosteric transformation which takes place in hemoglobin. Upon ligand dissociation, the heme undergoes an ultrafast doming which propagates along the whole protein. Such conformational changes involve many different time scales and much work is devoted to this problem. In order to investigate ultrafast conformational changes, we have set up a time-resolved circular dichroism experiment. Circular dichroism (CD: difference in absorption for a left and a right polarized light) is an optical characteristic of chiral molecules. In large molecules such as myoglobin, it relies on the spatial arrangement of the various chromophores. It therefore joins the advantages of optical techniques (among which is an unprecedented time resolution) with the ability to probe the response of the molecules on a global scale, contrarly to the usual techniques such as absorption or Raman scattering which are generally sensitive to local features. Time-resolved $\mathrm{CD}$ has been proposed and demonstrated in the past, ${ }^{1,2}$ but no convincing results were obtained, in particular, because the time resolution proved to be not good enough to yield interesting features.

Time-resolved CD experiments, however, suffer from a serious drawback which is the difficulty of interpretation. Indeed, CD being a nonlocal probe, it relies on many parameters because $\mathrm{CD}$ at a particular transition can be influenced by other transitions situated at distant part of the molecules. Furthermore, because CD is a signed quantity, large individual effects very often cancel each other out resulting in weak net signals.

The purpose of this article is to overcome these limita-

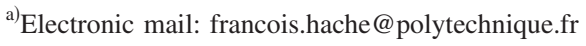

tions and to present a model calculation of $\mathrm{CD}$ in myoglobin permitting the interpretation of experimental results that we have obtained recently. ${ }^{3}$ In these experiments, a CD transient lasting about 100 ps was observed after photolysis of $\mathrm{MbCO}$. Here, we present a thorough analysis of the origin of the $\mathrm{CD}$ in $\mathrm{MbCO}$ and in the deoxyform $(\mathrm{Mb})$ which allows us to propose a mechanism responsible for this $\mathrm{CD}$ change.

The paper is organized as follows. In the next section, we summarize our main experimental results. In Sec. III, we present the model calculation we have used. This calculation is based on the polarizability theory which is well fitted for $\mathrm{CD}$ originating in the coupling of different chromophores in a large protein. This calculation is first applied to the steadystate geometries of the initial $(\mathrm{MbCO})$ and final $(\mathrm{Mb})$ states. Then, we simulate several intermediate configurations of the protein and calculate the corresponding CD spectra in order to single out the important parameters. Finally, in view of these calculations, we are able to propose an interpretation of our experimental results which is discussed in Sec. V.

\section{EXPERIMENT}

\section{A. Experimental details}

The time-resolved experiment is carried out on a $230 \mu \mathrm{M} \mathrm{MbCO}$ solution prepared by dissolving commercial horse heart metmyoglobin (Sigma) in a buffer (100 mM tris$\mathrm{HCl}, p \mathrm{H}=8.0$ ). The solution is placed in a $1 \mathrm{~mm}$ path-length silica cell. The experimental setup is based on a classical pump-probe experiment. The pump is directly obtained after frequency doubling a $1 \mathrm{kHz}$ titanium-sapphire output. The pump pulses have an energy of about $300 \mathrm{~nJ}$ and their duration is about 250 fs. $15 \%-20 \%$ of the MbCO molecules are photolyzed by the pump. The sample is continuously moved so that a fresh sample is available at each laser shot. The probe pulses, obtained by continuum generation in a sapphire plate, are tunable from 410 to $500 \mathrm{~nm}$. These pulses are sent 


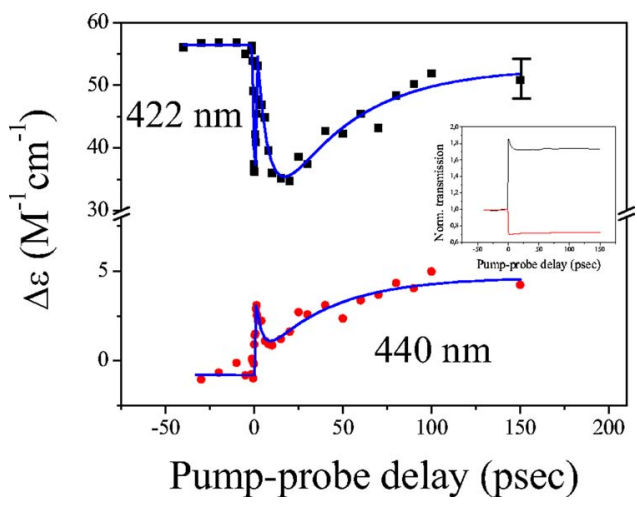

FIG. 1. Circular dichroism for $\lambda=422 \mathrm{~nm}$ (close to the MbCO peak) and $\lambda=440 \mathrm{~nm}$ (close to the Mb peak) as a function of the pump-probe delay. The CD curves are fitted by two exponential functions ( 7 and 43 psec). The inset shows the corresponding normalized transmission curves.

through the sample after a time delay and the transmitted energy is measured by a photomultiplier tube. In order to access the $\mathrm{CD}$, the probe polarization is modulated alternately left and right circular with a longitudinal Pockels cell on which we apply a $120 \mathrm{~Hz}$ square voltage. Due to $\mathrm{CD}$, this polarization modulation translates into a $\sim 10^{-3}$ transmission modulation which is detected by a lock-in amplifier synchronized with the Pockels cell. This setup allows us to simultaneously measure the transmission and the $\mathrm{CD}$ of our sample as a function of the delay between the pump and the probe. Even though the principle is straightforward, this experiment demands a very fine control of the beam polarization in order to avoid artifacts. ${ }^{4}$ The Pockels cell is very carefully aligned through a detailed procedure. ${ }^{5}$ Furthermore, using various configurations for the pump polarization allows artifacts to be further reduced.

\section{B. Results}

Time-resolved CD was first obtained on a nanosecond time scale for probe wavelengths spanning the Soret band. ${ }^{3}$ The striking feature of these experiments was that, on this time scale, no dynamics could be observed in the CD signal: the deoxy-myoglobin $\mathrm{CD}$ spectrum was immediately recovered, independently of the time delay. At wavelengths corresponding to the $\mathrm{MbCO}$ peak, we could only observe a downward step whereas at wavelengths corresponding to $\mathrm{Mb}$ peak, only an upward step was obtained. However, focusing on the very first times allowed us to observe a nice feature occurring in the CD spectrum on a $100 \mathrm{ps}$ time scale. The results for a probe wavelength of $422 \mathrm{~nm}$ (close to the MbCO peak) and of $440 \mathrm{~nm}$ (close to the $\mathrm{Mb}$ peak) are displayed in Fig. 1. The peak at zero delay is due to the pumpprobe overlap and does not yield conformational information. This peak corresponds to the pump-probe cross correlation and gives an estimate of our time resolution (650 fs). At positive delays, one gets a large CD drop on a $10 \mathrm{ps}$ time scale, followed by a relaxation toward the $\mathrm{Mb}$ steady state in 100 ps. Note that a similar trend was obtained for a wavelength of $355 \mathrm{~nm}$ in Ref. 2. In this article, the time resolution was not good enough to see the decrease, but an increase was observed on a $300 \mathrm{ps}$ time scale, in qualitative agreement with our work.

It is important to note at this point that this $100 \mathrm{ps}$ time scale allows us to rule out possible artifacts and to confidently assign this $\mathrm{CD}$ change to conformational issues. Purely electronic changes that occur in the heme after photolysis are very short lived ${ }^{6}$ and appear in our experiment as intantaneous. Temperature rise due to the absorption of energetic photons is not expected to perturb much electronic transitions and furthermore are known to be over in $20 \mathrm{ps.}^{7}$ Finally, pump-induced anisotropy which could deeply influence the $\mathrm{CD}$ would depend on the rotational diffusion time of myoglobin, measured to be $35 \mathrm{~ns}^{8}{ }^{8}$ This last point will be further discussed later on in view of our calculations.

Our experiment therefore discloses an ultrarapid conformational change which yields information on the very first steps of the allosteric mechanism in myoglobin. The following section of the article is devoted to presenting a model calculation of the $\mathrm{CD}$ which allows us to give an interpretation of this conformational change.

\section{CALCULATION OF THE CIRCULAR DICHROISM SPECTRA}

\section{A. Outline of the calculation}

The origin of the circular dichroism in the Soret band of carbon monoxy myoglobin has been identified by Woody and co-workers as principally coming from the coupling of the heme transitions with the $\pi-\pi^{*}$ transitions of the surrounding aromatic residues. ${ }^{9,10}$ We therefore chose to utilize the classical calculation introduced by DeVoe ${ }^{11}$ and further extended by Applequist. ${ }^{12}$ In particular, Applequist's treatment is very well fitted to the calculation of the chiroptical response of molecules consisting of several polarizable units arranged in a fixed position with respect to each other. The optical activity of such molecules originates in the interaction through dipole-dipole coupling of the different units.

Applequist's theory relies on the calculation of the electronic normal modes of the molecules. ${ }^{12}$ When expressed in the normal-mode basis, absorption and circular dichroism can be obtained readily. The main point is therefore the determination of these normal modes which implies a matrix diagonalization, a straightforward procedure (which can, however, be time consuming for large molecules). This calculation technique has the advantage that, although it is considerably simpler that most of the existing theories based on quantum chemistry, ${ }^{13}$ it nevertheless gives a very good description of CD spectra. In the case of myoglobin where the polarizable groups are very close, a point-charge description would be better and the CD spectra calculated with this simple technique will necessary be somewhat qualitative but anyway sufficient to obtain valuble information on the conformational issues under study.

We give here a brief account of the principles of the calculation. Details can be found in Refs. 12 and 14. Each molecule is supposed to be composed of $N$ polarizable units, indexed by subscript $i$. Each unit $i$ is in turn composed of several oscillators, indexed by subscript $s$. Introducing $\mathbf{u}_{i s}$ the 
unit vector describing the direction of the polarization of oscillator $i s$, the polarizability tensor of unit $i$ is

$$
\underline{\underline{\alpha}}_{i}=\sum_{s=1}^{p_{i}} a_{i s} \underline{\underline{u}}_{i s} \underline{u}_{i s} .
$$

The coefficients $a_{i s}$ are complex functions of the light frequency $\nu$ and are assigned a Lorentzian form,

$$
a_{i s}=\frac{c_{i s}}{\nu_{i s}^{2}-\nu^{2}-i \Gamma_{i s} \nu},
$$

$\nu_{i s}$ being the frequency of the oscillator and $\Gamma_{i s}$ its width. $c_{i s}$ is a coefficient proportional to the oscillator strength $\left(c_{i s}\right.$ $\left.=3 e^{2} f_{i s} / 4 \pi^{2} m\right)$.

Let us now consider the dipole moment $\mu_{i s}$ induced on oscillator $i s$ when one applies an electric field $\underline{E}$. Actually, the local electric field acting on the oscillator is is

$$
\underline{E}^{\mathrm{loc}}\left(u_{i s}\right)=\underline{E}-\sum_{j \neq i} \sum_{t} \underline{T}_{i j} \cdot \underline{\mu}_{j t}
$$

where the last term accounts for the dipolar field generated by the other units $j t$, with $\underline{\underline{T}}$ the symmetric dipole-dipole interaction tensor,

$$
\underline{\underline{T}}_{i j}=r_{i j}^{-3} \underline{\underline{I}}-3 r_{i j}^{-5} \underline{r}_{i j} \underline{r}_{i j} \text {. }
$$

Interactions between oscillators pertaining to the same unit are taken into account in the description of isolated groups and the matrix $\underline{\underline{T}}$ only deals with intergroup couplings. It is therefore sufficient to index $\underline{\underline{T}}$ by $i, j$ (without taking the $s, t$ subscripts into account). From Eqs. (3.1) and (3.3), one can introduce a symmetric interaction matrix $\underset{\equiv}{A}$,

$$
\stackrel{A}{\underline{\mu}} \underline{\underline{-}}=\underline{E} \text {. }
$$

Rows and columns of $\underset{=}{A}$ are designated by the double indices $i s$ and $j t$,

$$
A_{i s, j t}= \begin{cases}a_{i s}^{-1} \delta_{s t} & (i=j) \\ \underline{u}_{i s} \cdot \underline{\underline{T}}_{i j} \cdot \underline{u}_{j t} & (i \neq j) .\end{cases}
$$

Diagonalization of $A$ yields the normal modes of the ensemble of oscillators. Each normal mode is depicted by an eigenvector $\underline{t}^{(n)}$ whose elements $t_{i s}^{(n)}$ correspond to the contribution of the oscillator $i s$. With the help of these normal modes, one obtains quite easily the matrix elements of $\underline{\underline{B}}$ $=\underline{B}^{-1}$, the inverse of matrix $\underset{=}{A}:^{12}$

$$
B_{i s, j t}=\sum_{n=1}^{p} \frac{t_{i s}^{(n)} t_{j t}^{(n)}\left(\nu_{n}^{2}-\nu^{2}+i \nu \Gamma_{n}\right)}{\left(\nu_{n}^{2}-\nu^{2}\right)^{2}+\nu^{2} \Gamma_{n}^{2}} .
$$

In this expression, contrary to Applequist's calculation, we do not try to connect the $\Gamma_{n}$ 's with the oscillator widths $\Gamma_{i s}$ but rather consider them as phenomenological parameters. This feature seems reasonable as bandwidths are introduced to take into account the coupling of the transition with the environment and are therefore better defined for a normal mode than for an isolated transition. Besides this fundamental reason, it is also consistent with the current practice which consists to extract the $\Gamma_{n}$ 's from the width of the experimental absorption spectra. This matrix $\underline{B}$ allows the absorption and the CD spectra to be expressed as

$$
\begin{aligned}
& \varepsilon=\frac{10}{\ln 10} \frac{e^{2} N_{A}}{2 \pi \varepsilon_{0} m c} \sum_{n=1}^{p} \frac{\nu^{2} \Gamma_{n}}{\left(\nu_{n}^{2}-\nu^{2}\right)^{2}+\nu^{2} \Gamma_{n}^{2}} f^{(n)}, \\
& \Delta \varepsilon=\frac{80}{3 \ln 10} \frac{N_{A}}{\varepsilon_{0} c^{2} \hbar} \sum_{n=1}^{p} \frac{\nu^{3} \Gamma_{n}}{\left(\nu_{n}^{2}-\nu^{2}\right)^{2}+\nu^{2} \Gamma_{n}^{2}} R^{(n)},
\end{aligned}
$$

where $e$ and $m$ are the electron charge and mass, respectively, $c$ the light velocity, and $N_{A}$ the Avogadro number, with SI units except for $\varepsilon$ and $\Delta \varepsilon$ which are expressed in the usual $\mathrm{M}^{-1} \mathrm{~cm}^{-1}$; the refractive index is supposed to be equal to 1 . In these expressions, we have introduced the oscillator and rotational strengths for the $n$th normal mode which can be calculated as

$$
f^{(n)}=\frac{4 \pi^{2} m}{3 e^{2}} \sum_{i s, j t} t_{i s}^{(n)} t_{j t}^{(n)} \underline{u}_{i s} \cdot \underline{u}_{j t}
$$

and

$$
R^{(n)}=\frac{\pi^{2} \hbar}{2} \sum_{i s, j t} t_{i s}^{(n)} t_{j t}^{(n)} \underline{r}_{i j} \cdot\left[\underline{u}_{i s} \times \underline{u}_{j t}\right] .
$$

In the following, we will be interested in adjusting calculational parameters to experimental curves. When expressing the frequencies in $\mathrm{eV}$ and the rotational strengths in Debye-Bohr magneton (DBM), Eqs. (3.8) and (3.9) read

$$
\begin{aligned}
& \varepsilon=18200 \sum_{n=1}^{p} \frac{\nu^{2} \Gamma_{n}}{\left(\nu_{n}^{2}-\nu^{2}\right)^{2}+\nu^{2} \Gamma_{n}^{2}} f^{(n)} \quad\left(\mathrm{M}^{-1} \mathrm{~cm}^{-1}\right), \\
& \Delta \varepsilon=2.6 \sum_{n=1}^{p} \frac{\nu^{3} \Gamma_{n}}{\left(\nu_{n}^{2}-\nu^{2}\right)^{2}+\nu^{2} \Gamma_{n}^{2}} R^{(n)} \quad\left(\mathrm{M}^{-1} \mathrm{~cm}^{-1}\right) .
\end{aligned}
$$

The interesting feature of this calculation is that it allows the role of each component of the total rotational strength to be determined: the contribution of the coupling between groups $i$ and $j$ to the rotational strength of the $n$th normal mode is given by

$$
R^{(n)}(i, j)=\frac{\pi^{2} \hbar}{2} \sum_{s, t} t_{i s}^{(n)} t_{j t}^{(n)} \underline{r}_{i j} \cdot\left[\underline{u}_{i s} \times \underline{u}_{j t}\right] .
$$

\section{B. Steady-state CD spectra}

Myoglobin is a 153 amino acid protein which is composed of eight $\alpha$ helices, labeled A-H. These helices form a rather rigid structure which encompasses the so-called heme pocket. The heme cofactor is linked to the F helix through a bond between its central iron atom and the "proximal histidine" (His93). Ligands such as $\mathrm{O}_{2}$ or $\mathrm{CO}$ can reversibly bind to the iron atom. On the other side of the heme pocket lies the "distal histidine" (His64) (see Fig. 2). Steady-state structures of the protein in its liganded or deliganded forms have been measured by x-ray diffraction. ${ }^{15}$ These structures are available through the Protein Data Bank (MbCO: 1A6G, Mb: $1 \mathrm{~A} 6 \mathrm{~N})$. They are the starting point of our calculations.

Absorption and CD spectra of $\mathrm{MbCO}$ and $\mathrm{Mb}$ were measured with a Jobin-Yvon CD6 CD-spectrometer. The spectra 


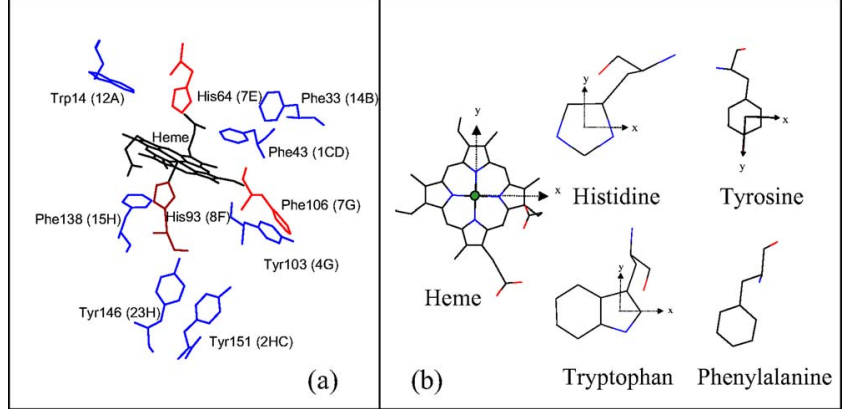

FIG. 2. (a) Sketch of the main aminoacids involved in the CD signal. The residues yielding a positive (resp. negative) rotational strength are drawn in blue (resp. red). The proximal histidine (His93) is singled out due to its particular role: it induces the lifting of the degeneracy of the heme transitions but contributes very little to the rotational strength. His 36, His97, Phe46, and Trp7 which are considered in the calculation are not represented because of their weak contribution to the rotational strength. (b) Sketch of the residues with their coordinate axes.

are displayed in Fig. 3. Our first task is to utilize our calculations to reproduce the experimental steady-state spectra.

\section{Choice of the parameters}

To reproduce the $\mathrm{CD}$ of $\mathrm{MbCO}$, we have considered the coupling of the heme with 14 aromatic residues close enough to interact efficiently. On top of the 12 residues already considered by Hsu and Woody, ${ }^{9}$ we have also introduced two tryptophans which are expected to give nonnegligible contributions $^{16}$ (see Tables II, III, and Fig. 2). Fitting of experimental curves with Eqs. (3.12) and (3.13) amounts to taking the protein geometry and calculating the normal modes for these 15 subunits (Heme+aromatic residues). Beside the geometry given by the $\mathrm{x}$-ray structures, this calculation requires knowledge of several parameters for the aromatic side chains, on the one hand, and for the heme transitions, on the other hand (see Fig. 2).

a. Aromatic residues. The coupling between polarizable units is more efficient if the transition energies are closer. The Soret band of the heme that we consider being in the visible, major contributions is expected from the low-energy electronic bands of the surrounding aromatic residues. Parameters describing these transitions were taken in the litera-

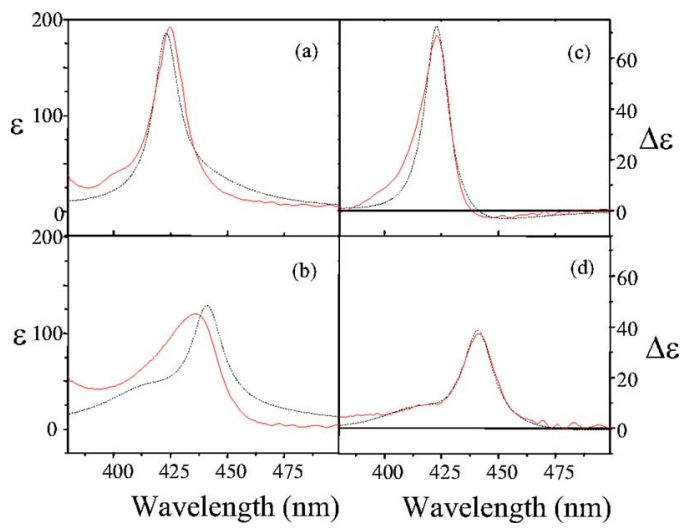

FIG. 3. Absorption spectra for (a) MbCO and (b) Mb; CD spectra for (c) $\mathrm{MbCO}$ and (d) Mb. The red solid lines are experimental spectra and the black dashed lines are calculated ones.
TABLE I. Energy, oscillator strength, and polarization of the electronic transitions of the aromatic pending groups. The polarization is expressed as the angle (in degrees) between the transition moment and the $x$ axis (see Fig. 2).

\begin{tabular}{|c|c|c|c|}
\hline Heme & Energy (eV) & Osc. str. & Polarization \\
\hline $\mathrm{MbCO}$ & 2.94 & 0.75 & $x, y$ \\
\hline \multirow[t]{2}{*}{$\mathrm{Mb}$} & 2.82 & 0.5 & $x, y$ \\
\hline & 3.00 & 0.4 & $x, y$ \\
\hline Amino acid/residue & Energy $(\mathrm{eV})$ & Osc. str. & Polarization \\
\hline Histidine/ & 5.10 & 0.12 & 58 \\
\hline \multirow[t]{3}{*}{ Imidazole } & 6.02 & 0.03 & -26 \\
\hline & 7.56 & 0.41 & 51 \\
\hline & 8.00 & 0.07 & -23 \\
\hline Tyrosine/ & 4.51 & 0.02 & $x$ \\
\hline \multirow[t]{3}{*}{ Phenol } & 5.82 & 0.13 & $y$ \\
\hline & 6.66 & 1.1 & $y$ \\
\hline & 6.66 & 1.1 & $x$ \\
\hline Tryptophan/ & 4.37 & 0.05 & 28 \\
\hline \multirow[t]{3}{*}{ Indole } & 4.77 & 0.12 & -41 \\
\hline & 6.02 & 0.6 & 23 \\
\hline & 6.35 & 0.5 & -33 \\
\hline Phenylalanine/ & 6.94 & 0.9 & $x$ \\
\hline Phenyl & 6.94 & 0.9 & $y$ \\
\hline
\end{tabular}

ture. In particular, an ab initio study of phenylalanine, tyrosine, and tryptophan was published recently ${ }^{17}$ and provided us with the required information. Things are, however, more complicated for histidine which plays a crucial role in the calculation given that the two closest amino acids from the heme are histidines. Complete information including the direction of the transition moments are not available and we have utilized Woody's data. ${ }^{9}$ We have also tried other CNDO results. ${ }^{18}$ Given the semiquantitative level of our calculation, the precise choice for histidine proved not to be determining. Altogether, for the 14 aromatic residues, a total of 46 transitions was considered. Summary of the parameters is given in Table I.

b. Heme. Considering $\mathrm{MbCO}$, the Soret band can be reproduced with a unique transition at $2.94 \mathrm{eV}$ which in reality corresponds to two heme transitions whose degeneracy is lifted by the coupling with the surrounding residues. We therefore adjusted the heme transition frequencies and oscillator strengths so as to reproduce correctly the absorption spectrum (see Table I). After coupling with the aromatic residues, the total Soret oscillator strength amounts to 1.35 , in agreement with the known value. ${ }^{19}$ These same parameters are then used to fit the CD spectrum. Bandwidths for the two transitions are also adjusted through these fitting procedures. The results of these fitting procedures are displayed in Fig. 3. The overall agreement is remarkable. In particular, Eqs. (3.12) and (3.13) were readily used with no other adjustment than the oscillator strength of the heme transitions and the bandwidths of the normal modes to obtain the correct magnitude for both the absorption and the $\mathrm{CD}$ spectra.

The case of $\mathrm{Mb}$ is more complex as the heme absorption band is much broader and asymmetric than in MbCO. Two 
features can explain this: the onset of the $N$ bands and the vibronic coupling. ${ }^{20}$ To account for this complex structure in a simple manner, we have considered two pairs of degenerate transitions for the heme in its deoxy state. The fitting procedures were the same as for MbCO by using Eqs. (3.12) and (3.13). As the agreement is less good than for $\mathrm{MbCO}$, we focus on the fit of the CD spectrum. The absorption spectrum is not very satisfactorily reproduced, indicating the inadequacy to account for the complex structure of the $\mathrm{Mb}$ absorption band. However, the following discussion being devoted to the $\mathrm{CD}$ spectra evolution, we think that this empirical fitting will be reliable enough.

\section{MbCO spectrum}

Let us first comment on the MbCO CD spectrum. The Soret band originates in the heme $B$ bands which give birth to two normal modes. Analyzing these normal modes in detail, one obtains that they principally originate in the coupling of the heme with the proximal histidine. Furthermore, as this coupling is weak, the eigenfunctions closely resemble those of the heme with occurrence of a small fraction of the other states. The principal effect of this coupling is the lifting of the degenracy. The two degenerate heme transitions do not couple the same way with the histidine because the imidazole plane is oriented almost along the heme $x$ direction (Fig. 2 ). The heme transition oriented along this $x$ direction therefore couples more efficiently with the histidine than the one oriented along the $y$ direction. We can now examine the rotational strength for these two normal modes and we obtain +0.9 and -0.5 DBM. These numbers are in good agreement with the experimental value of $0.5 \mathrm{DBM}$ measured for the Soret band. Because the two transitions are very close, the total rotational strength roughly results from the addition of these two values which yields 0.4 DBM. The occurrence of two CD bands of opposite signs explains the shape of the CD spectrum, which is narrower than the absorption one. The strong positive peak comes from the positive contribution whereas the negative contribution results in a narrowing of the peak and the onset of a small negative dip on the longwavelength side. Note that correct fit of the CD spectrum is obtained only if the two normal transitions are assigned different bandwidths (0.09 for the high-lying transition and $0.35 \mathrm{eV}$ for the low-lying one). Fitting of the CD spectrum is very sensitive to this asymmetry. We can connect this asymmetry to the above-mentioned difference in the coupling of the two heme transitions with the proximal histidine. Indeed, our calculation shows that the dipole-dipole coupling energy is more than one order of magnitude larger for the heme $x$ transition than for the $y$ one. Given that transition bandwidths are closely connected to the coupling with the bath, it seems normal that the low-energy transition which is more coupled to the environment has a larger bandwidth.

In order to analyze the origin of the Soret rotational strength, we rewrite Eq. (3.11) as

$$
R^{(n)}=\sum_{j \neq \text { heme }} R^{(n)}(\text { heme }, j)+\sum_{i, j \neq \text { heme }} R^{(n)}(i, j),
$$

and we keep only the first term defined from Eq. (3.14) as
TABLE II. Calculation of the contributions of the $\pi-\pi^{*}$ transitions of individual residues to the rotational strength of the heme in the Soret band for $\mathrm{MbCO}$ and $\mathrm{Mb}$.

\begin{tabular}{cccccc}
\hline \hline & \multicolumn{2}{c}{ MbCO } & & \multicolumn{2}{c}{$\mathrm{Mb}$} \\
\cline { 2 - 3 } \cline { 5 - 6 } Residue $^{\mathrm{a}}$ & Distance $^{\mathrm{b}}$ & Rot. str. $^{\mathrm{c}}$ & & Distance $^{\mathrm{b}}$ & Rot. str. $^{\mathrm{c}}$ \\
\hline His36 (1C) & 15.08 & -12 & & 15.12 & -7 \\
His64 (7E) & 5.64 & -125 & & 5.22 & -148 \\
His93 (8F) & 3.22 & 6 & & 3.66 & -25 \\
His97 (2FG) & 5.88 & 2 & & 6.04 & -27 \\
Phe33 (14B) & 10.24 & 108 & & 10.21 & 116 \\
Phe43 (1CD) & 6.14 & 31 & & 6.37 & -39 \\
Phe46 (4CD) & 10.04 & 18 & & 10.23 & 59 \\
Phe106 (7G) & 13.85 & -63 & & 13.84 & -50 \\
Phe138 (15H) & 9.44 & 81 & & 9.21 & 83 \\
Tyr103 (4G) & 10.94 & 102 & & 10.81 & 74 \\
Tyr146 (23H) & 10.93 & 87 & & 11.01 & 1 \\
Tyr151 (2HC) & 14.63 & 39 & & 12.82 & 50 \\
Trp7 (5A) & 20.26 & 14 & & 20.79 & -8 \\
Trp14 (12A) & 14.81 & 116 & & 14.57 & 84 \\
Total & & & 404 & & 163 \\
\hline
\end{tabular}

${ }^{\text {aThe amino acids are denoted following the usual abbrevations: His }}$ =histidine, Phe $=$ phenylalanine, Tyr=tyrosine, and Trp=tryptophan. The symbols in parentheses designate the residue position in the helices A, B,... ${ }^{\mathrm{b}}$ All distances are in Angstroms.

${ }^{\mathrm{c}}$ All rotational strengths are in $10^{-3}$ Debye-Bohr magnetons (DBM).

${ }^{\mathrm{d}}$ The difference between the total rotational strength and the sum of the individual residue contributions comes from the interresidue coupling.

$$
R^{(n)}(\text { heme }, j)=\frac{\pi^{2} \hbar}{2} \sum_{s, t} t_{\text {heme, }, s}^{(n)} t_{j t}^{(n)} \underline{r}_{\text {heme }, j} \cdot\left[\underline{u}_{\text {heme }, s} \times \underline{u}_{j t}\right] .
$$

This definition allows the contribution of the individual aromatic residues to the Soret rotational strength to be thoroughly analyzed. Neglecting the second term in Eq. (3.15) amounts to neglecting the residue-residue couplings which contribute indirectly to the total rotational strength through the normal modes. This error is, however, very small (see Table II).

Contributions of the various residues according to Eq. (3.16) are listed in Table II. Even though details of the contributions are very dependent on the protein geometry, these results are in qualitative agreement with Ref. 9. The most important residues are His64, Phe33, Tyr103, and Trp14. Several points are worth mentioning. First, the proximal histidine does not contribute much to the total rotational strength. Even though this residue is of paramount importance for defining the normal modes, the imidazole group position being almost perpendicular to the heme plane, it only gives a weak rotational strength. Second, most of the amino acids contribute a positive rotational strength. A notable exception is the distal histidine which gives a strong negative signal. Finally, note that all helices play a role but interestingly, the $E$ helix which encompasses the heme pocket gives a negative signal whereas the other, more distant helices contribute a positive signal.

\section{3. $M b$}

In $\mathrm{Mb}$, the Soret band is shifted to longer wavelengths and the major component peaks at $430 \mathrm{~nm}$. For this peak, 
TABLE III. Calculation of the contributions of the $\pi-\pi^{*}$ transitions of individual residues to the rotational strength (in $10^{-3} \mathrm{DBM}$ ) of the heme in the high-energy band of $\mathrm{Mb}, \mathrm{Mb}^{*}$, and $\mathrm{Mb}^{* *}$ (see text).

\begin{tabular}{cccc}
\hline \hline Residue & $\mathrm{Mb}^{*}$ & $\mathrm{Mb}^{* *}$ & $\mathrm{Mb}$ \\
\hline His36 (1C) & -10 & -3 & -7 \\
His64 (7E) & -5 & -77 & -25 \\
His93 (8F) & 7 & -9 & 9 \\
His97 (2FG) & 18 & 45 & 16 \\
Phe33 (14B) & 34 & 32 & 44 \\
Phe43 (1CD) & 164 & 13 & 89 \\
Phe46 (4CD) & -33 & -19 & -9 \\
Phe106 (7G) & -38 & -26 & -36 \\
Phe138 (15H) & 16 & 37 & 31 \\
Tyr103 (4G) & 48 & 32 & 46 \\
Tyr146 (23H) & 85 & 47 & 11 \\
Tyr151 (2HC) & 12 & 11 & 23 \\
Trp7 (5A) & 7 & -23 & -1 \\
Trp14 (12A) & 57 & 41 & 57 \\
Total & 363 & 101 & 246 \\
\hline \hline
\end{tabular}

which comes from the $B$ band of the heme, the same conclusions as for MbCO can be drawn (see Table II). These results are helpful to analyze the measurement taking place at $440 \mathrm{~nm}$. However, for $422 \mathrm{~nm}$, it is important to also consider the high-energy shoulder which relies on the higher heme transitions. Analysis of the individual contributions shows that the most important contribution to this highenergy part of the CD spectrum comes from Phe 43 whose benzene plane lies parallel to the heme plane (see Table III).

\section{SIMULATION OF THE EXPERIMENTS}

We want in this section to utilize our calculations to better understand the experimental signals that we have obtained (Fig. 1). Even though we know the starting and ending points $(\mathrm{MbCO}$ and $\mathrm{Mb})$, it is not sufficient to consider the corresponding steady-state $\mathrm{CD}$ spectra to understand our data. The first reason is that following the $\mathrm{CO}$ dissociation, the heme undergoes an instantaneous electronic transition from a low-spin state to a high-spin one. As already stated, this change is accompanied by a shift of the transition frequencies as well as a decrease of the oscillator strengths. Furthermore, the heme domes and the iron atom moves out of the heme plane by a fraction of an angstrom. ${ }^{21}$ This doming is responsible for the larger coupling between electronic and vibrational transitions. ${ }^{20}$ This change of electronic transitions automatically induces a change of $\mathrm{CD}$ which has nothing to do with a conformational change. It is therefore important to calculate the $\mathrm{CD}$ for intermediate configurations in order to single out the effects of conformational changes. In the following, we consider several relevant intermediate configurations. The predicted CD spectra are displayed in Fig. 4. Before proceeding to these calculations, we would like to comment on the effect of the $\mathrm{CO}$ molecule. $\mathrm{CO}$ has no transition in the visible or near ultraviolet and therefore does not couple efficiently with the heme electronic transitions. However, this weakness of the interaction can be counterbalanced by its proximity to the heme. When bound to the heme, the $\mathrm{CO}$ molecule stands in an upright position with

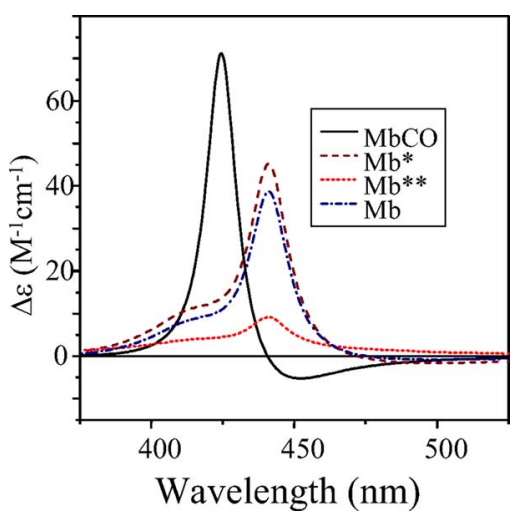

FIG. 4. Calculated spectra for $\mathrm{MbCO}$ and $\mathrm{Mb}$ and for two intermediate configurations $\mathrm{Mb}^{*}$ and $\mathrm{Mb}^{* *}$ (see text).

respect to the heme plane and consequently does not contribute to the MbCO CD. After leaving the heme iron, the $\mathrm{CO}$ rapidly goes to a first docking site where it stays hundreds of nanoseconds. $^{22}$ This docking site being $3 \AA$ away, the effect of $\mathrm{CO}$ on the $\mathrm{CD}$ spectrum is negligible. Finally, $\mathrm{CO}$ motion could impact the $\mathrm{CD}$ only in the very first picoseconds when going from the heme to its docking site. This time scale being out of reach of our experiment, we can safely neglect the contribution of $\mathrm{CO}$ to our experiment.

\section{A. Intermediate configurations}

As a first intermediate configuration, that we denote $\mathrm{Mb}^{*}$, we consider the case where the protein geometry is the same as MbCO but where the electronic state of the heme has been shifted to the $\mathrm{Mb}$ one. Even though this configuration has no real existence because the change in the electronic transitions and doming of the heme are closely connected, it serves as a starting point of the conformational changes after getting rid of the variation of the electronic state. The predicted $\mathrm{CD}$ change when going from $\mathrm{MbCO}$ to $\mathrm{Mb}^{*}$ corresponds to the instantaneous drop observed at $422 \mathrm{~nm}$ (near the MbCO peak) and to the instantaneous increase at $440 \mathrm{~nm}$ (near the Mb peak).

Following this very first step, we have simulated the effect of the change of geometry of the heme. We have therefore taken the geometry of $\mathrm{MbCO}$ for all the amino acids except for the heme. We have also considered the case where the heme and the proximal histidine have been moved to their final geometry. In both cases, the corresponding CD spectra are very close to the final Mb CD spectrum. This interesting feature shows that the large $\mathrm{CD}$ change that we observe in our experiment cannot be related to a progressive change from the $\mathrm{MbCO}$ geometry to the $\mathrm{Mb}$ one. We have therefore to examine other possible explanations of our observations. Given the importance of the proximal and distal histidine in the origin of the CD spectrum, we have examined the effects of a rotation of the imidazole planes on the CD spectra. Rotating the distal histidine, in fact, did not allow to lead a large change and even though such movements are probable due to collisions of the $\mathrm{CO}$ in the first docking site, they cannot be invoked to interpret our experiment. 


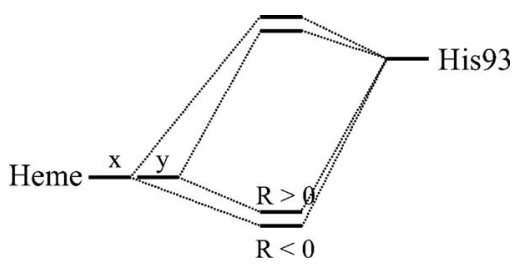

FIG. 5. Sketch of the electronic levels for the heme and His93 and the normal modes resulting from their coupling. The two low-lying normal modes have rotational strengths of opposite signs.

\section{B. Rotation of the proximal histidine}

Rotation of the proximal histidine about the axis perpendicular to the heme proved to deeply affect the CD spectrum. This result of our calculation is surprising at first sight because, as stated previously, the proximal histidine does not provide a large contribution to the total rotational strength due to its perpendicular position with respect to the heme. Indeed, rotation of the imidazole plane does not change this symmetry. The reason for the huge effect of this rotation is to be sought in the coupling between the heme and the proximal histidine which mainly determines the normal modes of the system. As schematically drawn in Fig. 5, the two $x, y$ degenerate heme transitions are coupled to His93 and give birth to two nondegenerate normal modes. Each mode is a combination of all the original transitions, with a predominance of the heme transitions, and has a rotational strength which originates in the coupling of the heme with the other residues. In $\mathrm{MbCO}$ or $\mathrm{Mb}$, the two modes have rotational strengths with opposite signs, giving rise to the CD spectrum. When His93 is rotated, the positions and the oscillator strengths of the normal modes do not change appreciably but their composition in terms of original transitions is strongly modified and so is the repartition of the rotational strength between the two modes. This change translates into a large modification of the CD spectrum. Note that the contribution of the proximal histidine to the total rotational strength does not change dramatically and remains very small. ${ }^{9}$ The change comes from the other amino acids. We have considered a rotation of $\pi / 6$ in Fig. 4. One can see that the CD decreases strongly in this case. Rotating further the proximal histidine results in an interchange of the sign of the rotational strength between the two normal modes. It is not clear how bandwidths should be modified in such a strong perturbation, but in any case, such a rotation is expected to completely change the shape of the CD spectrum. The configuration with the proximal histidine tilted by $\pi / 6$ is denoted $\mathrm{Mb}^{* *}$ in the following.

\section{Pump-induced anisotropic CD}

We have taken advantage of our calculation to address another problem that can be encountered in our timeresolved $\mathrm{CD}$ experiment, namely, the pump-induced anisotropy of the sample. Indeed, myoglobin molecules whose heme plane lies parallel to the pump polarization are preferentially excited. This means that after excitation, the sample is no longer isotropically distributed. $\mathrm{CD}$ in anisotropic samples being different from isotropic sample, ${ }^{23}$ could give rise to a time evolution of our $\mathrm{CD}$ signal connected to the

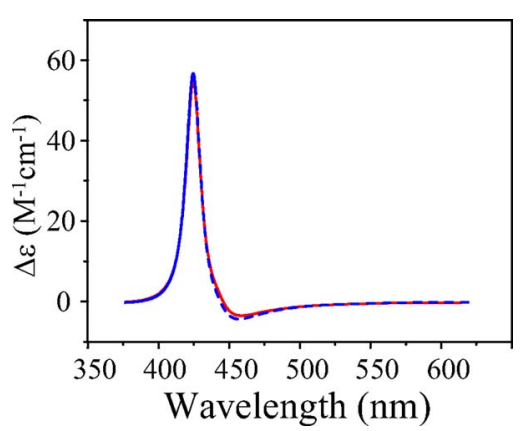

FIG. 6. Calculated CD spectra for a mixing of $80 \% \mathrm{MbCO}$ and $20 \% \mathrm{Mb}^{*}$ for an isotropic distribution (blue dashed line) and a pump-induced anisotropy (red solid line).

randomization of the sample and not to conformational changes. Even though such a reorientation process is expected to last $35 \mathrm{~ns}^{8}$ and therefore cannot explain our $100 \mathrm{ps}$ change, it seems interesting to quantify this effect. In a recent paper, ${ }^{14}$ we have extended Applequist's calculation to this anisotropy problem and shown that it was possible to account for anisotropy by introducing a tensorial rotational strength. We have applied this to our experiment by calculating the CD spectra for a sample where $20 \%$ of the molecules are excited and comparing the cases of a pump-induced anisotropic distribution and of an isotropic one. In the anisotropic case, the proportion of excited molecules depends on the angle between the normal to the heme and the beam propagation direction. Because it is proportional to the absorption cross section, it is readily calculated and averaged over the initial isotropic distribution. The results are displayed in Fig. 6, clearly demonstrating that this effect is negligible in our experiment. This insensitivity to the anisotropy of the distribution is connected to the $x, y$ quasidegeneracy of the two Soret transitions.

\section{DISCUSSION}

We come now to a discussion of our experimental results in view of our calculation. First of all, we can understand that no dynamics could be observed on nanosecond or longer time scale even though motion of remote amino acids is still expected on such time scales. ${ }^{24}$ Indeed, our calculation shows that once the electronic-induced change of the $\mathrm{CD}$ is taken into account, no major changes occur when passing from the liganded geometry to the deliganded one. Two points can explain this insensitivity. On the one hand, the residues move relatively little compared to the heme and on the other hand, even though individual residues can bring a noticeable change to the total rotational strength, these changes can be positive or negative and result on average in a weak modification.

The second point we want to address is the dip we observe in the time-resolved CD curves at 422 and $440 \mathrm{~nm}$. As indicated in Sec. IV, the only geometric change that can induce such a decrease of the CD is connected to the rotation of the imidazole plane in the proximal histidine. Note that this feature allows us to understand the observed trends for $422 \mathrm{~nm}$ as well as for $440 \mathrm{~nm}$. In order to characterize better this effect, we have analyzed the contributions of the indi- 


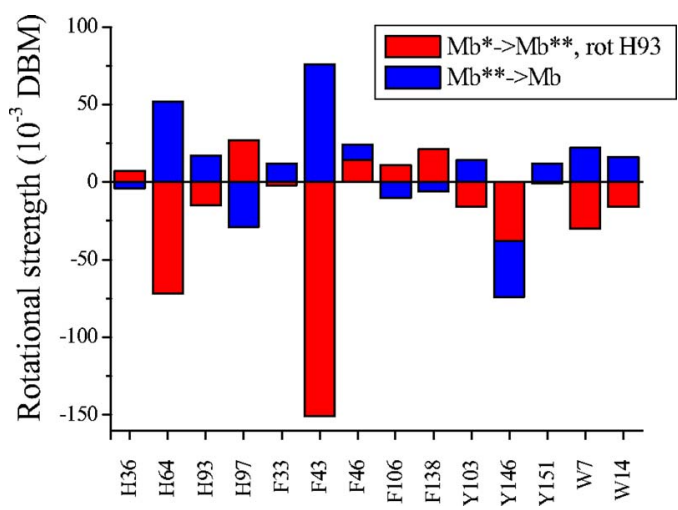

FIG. 7. Histogram of the calculated variation of the contribution of the residues to the rotational strength of the high-energy band when passing from $\mathrm{Mb}^{*}$ to $\mathrm{Mb}^{\text {** }}$ (red) and from $\mathrm{Mb}^{\text {*** }}$ to $\mathrm{Mb}$ (blue). See text for the definition of the intermediate structures.

vidual residues to the rotational strength (Table II) and we have plotted in Fig. 7 their variation when the protein passes from $\mathrm{Mb}^{*}$ to $\mathrm{Mb}^{* *}$ and from $\mathrm{Mb}^{* *}$ to $\mathrm{Mb}$. It is clear from this figure that rotation of the proximal histidine profoundly changes the contribution of His64 and Phe43 to the total rotational strength. These two amino acids are indeed very close to the heme and strongly coupled to it. Upon relaxation of the proximal histidine together with the rest of the protein toward the final deoxy configuration, evolution of the rotational strengths is reversed, canceling the major part of the effect. Only for Tyr146 does the contributions evolve in the same direction, but as already stated, this noticeable change is almost completely canceled out by other contributions.

Our studies therefore allow us to propose a picture of what is happening in the first 100 ps following myoglobin photolysis. Following the departure of the $\mathrm{CO}$, the heme undergoes a very rapid doming which in turn creates a stress on the proximal histidine. From our measurements, this stress settles in a few tens of picoseconds. This is the stress which is responsible for the large change in the $\mathrm{CD}$ that we observe. It is not possible to know precisely from our measurements what is the motion of the histidine as there exist many degrees of freedom, but the role of histidine in the very first step of the process can be confidently assessed from our calculations. This stress then relaxes through the movement of the F helix to which the proximal histidine belongs which in turn triggers the whole relaxation of the protein to its deoxy form. From our measurements, we see that the histidine stress relaxes on a $100 \mathrm{ps}$ time scale, revealing an important step in the heme pocket relaxation. It is interesting to note that these time scales for the rise and for the decay of the signal are in agreement with the energetic dynamics measured through thermal phase grating. ${ }^{25}$ This feature conforms with the idea that local motions are directly connected to energetic relaxation in the bath. These times are moreover similar to the dynamics of the frequency shift of the Fe-His vibration measured by time-resolved resonance Raman spectroscopy. ${ }^{26}$ They could also be associated with the slow components already observed in the heme doming. ${ }^{27}$

\section{CONCLUSION}

In this article, we have presented a model calculation of the CD spectrum of myoglobin. By calculating the spectrum for various configurations, we have been able to show that global change of the protein geometry from the initial state $(\mathrm{MbCO})$ to the final one $(\mathrm{Mb})$ does not explain the transient decrease that we observe in our time-resolved experiment. However, our calculation pinpoints the crucial role played by the proximal histidine in defining the protein normal modes and shows that motion of this histidine can explain our results. We can therefore conclude that our CD experiment allows us to observe the initial stress of the proximal histidine following the heme doming. This stress relaxes through the movement of the F helix and triggers the global rearrangement of the myoglobin to its deoxy configuration. Relaxation of the proximal histidine is measured on a $100 \mathrm{ps}$ time scale. This short time scale corresponds to the very first motion of the protein. By accessing such a short time scale, this time-resolved technique is very complementary to timeresolved x-ray diffraction techniques which yield information on a 150 ps time scale. ${ }^{24}$ This technique should also be very powerful in studying first events in $\alpha$-helix formation because such secondary structures have characteristic CD signatures in the ultraviolet that should be accessible to timeresolved experiments.

\section{ACKNOWLEDGMENT}

We thank Alexandre Chenal for providing us access to a CD spectrometer for the measurement of the steady-state CD spectra.

${ }^{1}$ S. J. Milder, S. C. Bjorling, I. D. Kuntz, and D. S. Kliger, Biophys. J. 53, 659 (1988).

${ }^{2}$ X. Xie and J. D. Simon, Biochemistry 30, 3682 (1991).

${ }^{3}$ T. Dartigalongue and F. Hache, Chem. Phys. Lett. 415, 313 (2005).

${ }^{4}$ J. W. Lewis, R. A. Golbeck, D. S. Kliger, X. Xie, R. C. Dunn, and J. D. Simon, J. Phys. Chem. 96, 5243 (1992).

${ }^{5}$ T. Dartigalongue and F. Hache, J. Opt. Soc. Am. B 20, 1780 (2003).

${ }^{6}$ S. Franzen, L. Kiger, C. Poyart, and J. L. Martin, Biophys. J. 80, 2372 (2001).

${ }^{7}$ M. Lim, T. A. Jackson, and P. A. Anfinrud, J. Phys. Chem. 100, 12043 (1996).

${ }^{8}$ J. Hofrichter, A. Ansari, C. M. Jones, R. M. Deutsch, J. H. Sommer, and E. R. Henry, Methods Enzymol. 232, 387 (1994).

${ }^{9}$ M. C. Hsu and R. W. Woody, J. Am. Chem. Soc. 93, 3515 (1971).

${ }^{10}$ G. Blauer, N. Sreerama, and R. W. Woody, Biochemistry 32, 6674 (1993).

${ }^{11}$ H. J. DeVoe, Chem. Phys. 43, 3199 (1965).

${ }^{12}$ J. Applequist, K. R. Sundberg, M. L. Olson, and L. C. Weiss, J. Chem. Phys. 70, 1240 (1979).

${ }^{13}$ C. Diedrich and S. Grimme, J. Phys. Chem. A 107, 2524 (2003).

${ }^{14}$ F. Hache and T. Dartigalongue, Chem. Phys. 303, 197 (2004).

${ }^{15}$ J. Vojtechovsky, K. Chu, J. Berendzen, R. M. Sweet, and I. Schlichting, Biophys. J. 77, 2153 (1999).

${ }^{16}$ G. Geraci and L. J. Parkhurst, Methods Enzymol. 76, 262 (1981).

${ }^{17}$ D. M. Rogers and J. D. Hirst, J. Phys. Chem. A 107, 11191 (2003).

${ }^{18} \mathrm{C}$. Andraud (Private communication).

${ }^{19}$ W. A. Eaton and J. Hofrichter, Methods Enzymol. 76, 175 (1981).

${ }^{20}$ A. Cupane, M. Leone, E. Vitrano, L. Cordone, U. R. Hiltpold, K. H. Winterhalter, W. Yu, and E. E. Di Iorio, Biophys. J. 65, 2461 (1993).

${ }^{21}$ C. Rovira, K. Kunc, J. Hutter, P. Ballone, and M. Parrinello, J. Phys. Chem. A 101, 8914 (1997).

${ }^{22}$ D. Vitkup, G. A. Petsko, and M. Karplus, Nat. Struct. Biol. 4, 202 (1997). 
${ }^{23}$ D. Che, R. A. Goldbeck, and D. S. Kliger, J. Chem. Phys. 100, 8602 (1994).

${ }^{24}$ F. Schotte, M. Lim, T. A. Jackson, A. V. Smirnov, J. Solman, J. L. Olson, G. N. Phillips, M. Wulff, and P. A. Anfinrud, Science 300, 1944 (2003).
${ }^{25}$ R. J. D. Miller, Acc. Chem. Res. 27, 145 (1994).

${ }^{26}$ Y. Mizutani and T. Kitagawa, J. Phys. Chem. B 105, 10992 (2001).

${ }^{27}$ M. Lim, T. A. Jackson, and P. A. Anfinrud, Proc. Natl. Acad. Sci. U.S.A. 90, 5801 (1993). 